\title{
Considerations for Design of Studies of Normal Aging, Accelerated Aging, and Neurodegeneration
}

\begin{abstract}
Introduction
In pursuit of our goal to provide guidelines for studies across the breadth of the neuroscience field, the Journal of Neuroscience is publishing a series of editorials on experimental design aimed at increasing transparency and reproducibility of published findings. In this editorial, we discuss experimental considerations particularly important for studies of healthy aging and neurodegeneration in human subjects, rodents, invertebrates, and in cultured cells.
\end{abstract}

\section{Considerations for studies of normal aging in human subjects and model organisms}

As a general point, it should be clear in the manuscript title what organism or model system is being studied. It is also important to define what is meant by "healthy" aging. Is normal aging being compared with a pathological condition? If so, it is helpful to operationalize what is meant by the "healthy" state. Since there are clear sex differences in various aspects of age-related changes, the sex of subjects in the study should always be reported and included as a variable. What is often missing in aging studies is measurement of unaffected phenotypes, as a way to show specific age dependence of the metrics of interest. Attributing a difference in a parameter across different ages can only be considered specific if there are also measurements (e.g., cellular functions, physiological or sensory processes or behaviors) that are unaffected. To determine that a particular phenotype is altered specifically during aging, a statistical interaction with a phenotype that is not altered is the most rigorous comparison. For example, in human neuroimaging studies, the cardiovascular response is known to change with age, making specific conclusions about hemodynamic differences between a younger and an older cohort of participants in a single brain region quite weak; and without affirmative evidence to the contrary, such conclusions are likely to be nonspecific.

The most critical decision for aging studies is what ages or time points are chosen for the study. For example, a study that compares young animals to old animals without middle-aged groups would be analogous to a human study that compared adolescents to elderly adults, without including a group of fully adult individuals. Without middle-age groups, it is unclear whether differences between young and old individuals reflect the aging process or are related to maturation. For example, the expression of a gene may decrease from young to middle age and thereafter remain stable or decrease. In general, more than two time points are necessary to make conclusions about effects of aging, and a larger number of time points is often more valuable.

A related point that is conceptually more challenging is that human studies using cross-sectional comparisons to make inferences about longitudinal changes during aging can be confounded by many factors and should be avoided whenever possible. For example, "aged" baby boomer brains may be very different from the brains of "young" college student cohorts born in the early 2000 s for reasons that have little to do with cellular- or systems-level senescence and could reflect differences in nutritional availability or exposure to environmental toxins during critical periods (as an example, scarcity during or after World War II vs abundant fast food in the 2000s) at different times, among many possibilities. Similarly, it is important to identify initial or primary changes due to aging or pathology, and to distinguish these from compensatory or maladaptive changes that emerge during the course of progressive neurodegeneration. In clinical studies, this might be achieved by considering longitudinal approaches that include at-risk individuals for the disorder being studied.

While in vitro systems can be informative for elucidating molecular mechanisms contributing to in vivo changes, using cell cultures alone to study normal aging can be problematic because of the short time periods (typically weeks) during which cultures are studied, relative to the lifespan of the species, and because of the artificial environment in which the cells are studied. Cultured neurons are useful for investigating mechanisms of neuroplasticity, cell degeneration, and survival but are most valuable when linked to observations of the in vivo impact of the genes, molecules, or pathways being tested. A common example would be behavioral and/or physiological studies of a transgenic animal combined with in vitro exploration of the effect of the genetic manipulation on circuits, cells, or molecules of interest.

\section{Mechanisms of normal aging and age-related neurobiological disorders}

A primary goal of JNeurosci is to publish studies that advance the knowledge of mechanisms underlying normal or pathological neurobiological processes. When designing studies on the neurobiology of aging, it is important to define what mechanisms are being evaluated. The first step is to determine the level at which you define the mechanism (whether molecular, cellular, systems, behavior) and then demonstrate the changes that are measured at that level. Specifically, what are the physiological or cognitive processes that are being investigated, and how do these change with intervention? In general, while broad molecular evaluations at the genomic, transcriptomic, or proteomic levels, or imaging studies, such as structural or resting state connectivity, are extremely valuable for describing all possible changes that can occur during normal or pathological aging, these studies are not considered mechanistic without some functional validation of the observed changes. Functional studies can be useful in determining what molecular differences cause downstream alterations, and for identifying those changes that contribute to pathology, or simply occur in tandem with pathology. Similarly, showing that a new pharmacological compound has an effect in vitro in animals or in humans would not be considered mechanistic without insight into the underlying mechanism, for example, a description 
of the compound's targets and their roles in a neurobiological process of interest.

For in vitro studies, selecting good models is very important. For example, experiments in immortalized cell lines alone may reflect processes that do not occur in vivo. Cell lines lose genetic material over time, can have aberrant patterns of division and growth, and are differentially sensitive to toxic compounds compared with unmanipulated neurons or glial cells. In many cases, the use of primary neuronal cultures relevant to the degenerative process is preferred (i.e., hippocampus or basal forebrain for Alzheimer's disease, motor neurons for ALS, or sensory cells for age-dependent sensory loss). In studies using primary cultures, specification of the cell types in the culture is necessary for interpretation of outcomes. Ultimately, validation of in vitro findings in an in vivo system can significantly strengthen the conclusions of the study. For studies of cellular mechanisms of neurodegeneration, demonstration of in vivo relevance is particularly important. For example, showing that a genetic mutation associated with Parkinson's disease alters survival of cells in culture does not guarantee that the change would result in neurodegeneration in vivo. Conversely, alterations in cellular phenotype in cultured cells may be more modest than effects in vivo. For example, a small change in the function of olfactory neurons or cochlear hair cells may lead to large changes in behavioral performance, given striking associations between sensory loss and cognitive decline.

\section{Animal models of human neurodegenerative disease}

Rodent models of human disease have been invaluable in identifying the function of genetic modifications that lead to neurodegeneration, as well as molecular pathways, cellular dysfunction, and systems-level alterations that contribute to disease progression or therapeutic response; however, no single rodent model recapitulates all aspects of these complex human disorders. When possible, testing a hypothesis in more than one disease model can strengthen the conclusions of a study. Further, there are likely to be interactions between disease-causing mutations and processes of normal aging, so studying the phenotypes of genetic alterations in young animals may not be sufficient to understand the mechanisms that lead to neuronal loss in human patients. Similarly, the value of studies in which neurodegeneration is induced by drugs can be limited, particularly when they involve healthy, young animals with acute damage. A genetic model with a wellestablished behavioral phenotype that recapitulates the disease can be very useful for comparison or validation.

When using animal models to propose a novel mechanism of neurodegeneration or neuroprotection, it is helpful to have a functional readout of the neuronal change being studied, such as a systems-level or behavioral output, that can help demonstrate the functional significance of the mechanism being studied. For each model, an appropriate behavioral test would either recapitulate the outcome of the human disease (e.g., motor dysfunction for ALS or hearing loss for cochlear degeneration) or relate to the normal rodent function of the brain area being studied (e.g., contextual learning for the hippocampus or altered metabolism for hypothalamic dysregulation). In all cases, just as we have emphasized in our editorial on behavioral studies in model organisms (https://www.jneurosci.org/collection/experimentaldesign-editorials), it is essential to power the study adequately to make a firm conclusion. This can be done by a priori determination of power based on pilot or published studies, or ideally, by internal replication to demonstrate that a significant novel outcome can be observed again when the same laboratory repeats it under similar conditions.

\section{Aging and neurodegeneration studies in invertebrate model organisms}

Both Caenorhabditis elegans and Drosophila melanogaster have been valuable for establishing fundamental mechanisms of cellular and organismal aging, as well as of neurodegeneration. JNeuroscivalues and encourages submissions based on these and other model organisms. As for rodent studies, it is important for invertebrate studies to address issues related to genetic background or selection of a particular model system. For studies of aging in short-lived invertebrates and other animals, investigators are encouraged to adopt a longitudinal design. If this is not practical, it is important to provide a rationale for the particular ages under study and indicate whether they correspond to immature or mature life stages.

\section{Conclusions}

The primary considerations of rigorous experimental design hold for studies of aging just as they do for all studies of neurobiological problems (https://www.jneurosci.org/collection/experimentaldesign-editorials). Attention to issues of statistical power and in vivo validation of findings from cell culture or bioinformatics studies using functional outcomes are shared across domains of neuroscience research. However, additional considerations affect our ability to draw strong conclusions about mechanisms of aging. For example, it is critical to be aware of how age-related peripheral changes can affect central functions. Because defective genes are carried by many inbred mouse lines, sensory aging, such as age-related hearing or vision loss, can occur at strain-specific rates, with possible downstream effects on brain function and behavior. Most important, studies of aging should include multiple age groups (e.g., young adult, middle aged, and old, at a minimum), and this consideration applies to experiments at all levels and in models, including cell cultures, invertebrates, rodents, healthy human subjects, and those with neurodegenerative disorders.

The Editorial Board of The Journal of Neuroscience https://doi.org/10.1523/JNEUROSCI.1527-19.2019 